\title{
Formación del profesorado universitario: mejorar a los docentes para mejorar la docencia
}

Miguel Zabalza Beraza*

\section{Resumen}

Tomando como marco de referencia el compromiso de la OCDE, "Teachers Matters: attracting, developing and retaining effective teachers", este artículo parte de tres ideas básica: la enseñanza universitaria es una actividad compleja; lograr una enseñanza de calidad requiere de un profesorado cualificado; para serlo se precisa de formación. Para combinar el compromiso de una docencia de calidad con la formación del profesorado, el texto plantea en primer lugar la "importancia del profesorado" como pieza clave en el desarrollo de una docencia de calidad. En un segundo apartado se analizan la evolución de la figura del docente y los nuevos requisitos del rol. Se mencionan, entre ellos, el desafío de la doble profesionalidad, las competencias didácticas, la implicación institucional y la apertura a la globalización y el trabajo en red. En tercer lugar, se analizan los procesos de formación del profesorado y los cambios que precisan en este nuevo recorrido: una formación orientada al desarrollo práctico de procesos y a la "acomodación" piagetiana, que sea capaz de dar respuesta tanto a las demandas técnicas de los docentes como a sus demandas psicosociales y que se plantee como un proceso de desarrollo a lo largo de la vida que los docentes puedan rentabilizar académicamente.

Palabras clave: docencia universitaria, formación del profesorado universitario, modelos de desarrollo docente.

\section{Higher education teacher training: qualify professors to qualify teaching}

\section{Abstract}

Taking as reference the commitment of the OECD: "Teachers Matters: attracting, developing and retaining effective teachers", this article departs from three basic assumptions: teaching at Higher Education is a very complex activity; to achieving quality in Higher Education requires a very qualified teacher; becoming a qualified teacher requires good education. To combine the commitment to quality teaching with teacher training, the text proposes, first, the "importance of teachers" as a cornerstone in the development of quality teaching. The second section analyzes the evolution of the teacher role and its new requirements, including the challenge

\footnotetext{
* Profesor de la Universidad de Santiago de Compostela, Departamento de Currículo y Organización Escolar. Santiago de Compostela, España.
} 


\section{Miguel Zabalza Beraza}

of the double professionalism, the teaching competencies, the institutional involvement and the openness to globalization and networking. Third, we analyze the processes of teacher education and some changes they need in this new route map: an education oriented to the practical development of processes and to the piagetian "accommodation" approach, allowing for responding to both the technical and the psychosocial demands of teachers, which is assumed as lifelong career development.

Keywords: higher education teaching, higher education teacher training, models of teacher education.

\section{Introducción}

Este texto trata de proponer una serie de reflexiones sobre la formación del profesorado (inicial y en servicio) en el nuevo contexto de lo que se ha dado en llamar el "proceso de convergencia europea" hacia un "Espacio Europeo de Educación Superior (EEES)". No pretendo hacer una revisión comparada en torno a los sistemas formativos en los diferentes países. Esa información está disponible en múltiples libros e informes que han ido apareciendo en los últimos años. ${ }^{1}$ Mi intención es repasar algunos de los grandes dilemas en los que se debate la formación de profesores en el momento actual y algunos de los retos que deberá afrontar en el futuro inmediato.

Al hablar de "formación de profesores" me estoy refiriendo a intervenciones de diversa índole y con diverso grado de permanencia: desde la formación inicial del profesorado que pretende acceder a la enseñanza universitaria hasta la formación continua del profesorado con experiencia (formación permanente, maestrías, doctorados, etc.), incluyendo, igualmente, las más recientes modalidades de grupos de innovación, redes profesionales, programas de formación en Facultades y Departamentos, etc. ¿Qué modelo de profesor subyace a todas esas iniciativas?

La construcción social del papel del profesor (incluso de su función instructiva y de la forma en que debería ejercerla) no es ajena, no puede serlo, a la naturaleza y sentido social que en cada periodo histórico se otorgue a la universidad. Proceso que viene condicionado por los propios cambios que, durante estos años, se han producido en el papel y la función social de las instituciones de Educación Superior. Podríamos decir, incluso, que es un cambio que se está produciendo a bandazos, al socaire de los vientos políticos que en cada momento mueven las velas de la nave educativa. El caso español puede ser un arquetipo de ese oleaje, con un tejer y destejer constante de políticas universitarias, como en el mito de la Penélope griega. Somos un buen ejemplo de cómo la falta de un consenso político básico sobre el papel de la educación y la función del sistema formativo, mantiene una constante inestabilidad en la estructura del sistema y dificulta enormemente la configuración de un perfil 
institucional y profesional coherente con dicha misión.

Esta reestructuración de las políticas universitarias ha generado un terremoto tan potente como el reciente de Fukushima. Y, como sucedió allí, la crisis económica ha provocado un tsunami complementario del que, según algunos, nos será difícil salir en bastantes años. Ciertamente, no resulta fácil hacer coincidir el desafío de la reestructuración institucional de las Universidades con la mayor crisis económica a la que nos hemos tenido que enfrentar en los últimos años.

Por eso me ha parecido especialmente estimulante el reto que, a nivel de profesorado en general, se ha propuesto la OCDE, bajo el lema "Europa necesita profesores" y que se ha concretado en todo un abanico de sugerencias a través del texto "Teachers Matters: attracting, developing and retaining effective teachers". ${ }^{2}$ La Revista de Educación, editada por el Ministerio de Educación español le dedicó un número monográfico ( $n^{\circ}$ 340, mayo-agosto 2006) bajo el subtítulo de La tarea de enseñar: atraer, formar, retener y desarrollar buen profesorado. Porque parece, efectivamente, que no sólo Europa, sino los países en general necesitan contar con un profesorado de calidad capaz de desempeñar las complejas funciones que hoy se atribuyen a las escuelas y los docentes, sea cual sea el nivel educativo en el que trabajan. Para las Universidades, atraer, formar, retener y desarrollar un profesorado competente, y hacerlo en contexto económico no favorable, va a ser uno de los desafíos fundamentales para el siglo que iniciamos.

Se podría decir, con justeza, que el mundo se mueve a nuestros pies, que están cambiando los referentes que dirigían nuestra acción de profesores y que, por tanto, ahora más que nunca, estamos necesitados de nuevos marcos que orienten la acción y estimulen el esfuerzo de quienes nos dedicamos a la docencia. En la Universidad han cambiado nuestros estudiantes, ha cambiado el mundo del conocimiento, han cambiado las demandas de los empleadores, han cambiado los procesos de transformación social y las necesidades de sus agentes, han cambiado las políticas y las fuentes de financiación, ha cambiado la cultura. Es en estas circunstancias donde cobra sentido aquella sentencia de Einstein: "Stupidity is doing the same things the same way and still expect differents results". Algo tiene que cambiar en la Universidad. Y serán insuficientes los cambios que hagamos hacia dentro de las instituciones (curriculares, organizativos, de estructura de los estudios) porque el gran cambio es hacia fuera. Lo que tiene que cambiar es la relación de la universidad con la sociedad que la mantiene de forma que sea capaz de responder a sus necesidades, a sus nuevos desafíos, a las demandas de los diversos agentes sociales. La universidad monasterio cerrada sobre sí misma, generando su propia cultura, viviendo de sus propios rituales y tradiciones ha desaparecido o está a punto de hacerlo. Se precisa de otro tipo de institución más abierta y flexible, más inserta en el mundo que le rodea (que cada vez más es el mundo global), más generosa con quienes acuden a ella desde situaciones personales y sociales divergentes (me- 


\section{Miguel Zabalza Beraza}

nos elitista), más rentable socialmente.

Pues bien, en esta situación cambiante, ¿cuál es el papel de las instituciones de Educación Superior? Y yendo a nuestro tema, ¿cuál es la función de los profesores y profesoras que trabajan en ellas? Soy consciente de que éste es un tema con muchos flancos y que precisaría de amplias reflexiones. No pretendo, ni sería capaz de llegar a tanto. Mi propósito aquí no es otro que el de provocar algo de debate e intercambio de ideas sobre la formación del profesorado universitario en un mundo en cambio. Insistiré, por tanto, en aquellos puntos que suponen, en mi opinión una importante modificación en los actuales planteamientos. Espero que, aunque simple, esta reflexión nos ayude a repensar esta cuestión. Este texto abordará los siguientes puntos:

I- La importancia del profesorado (vinculada a la importancia de la docencia).

II - La evolución de la figura del profesor.

III - La estructura de la formación y su evolución.

\section{I) La importancia del profesorado}

En el centro de la mejora escolar está la preocupación por mejorar la calidad de la enseñanza y el aprendizaje en el aula. Algunos estudios sobre las escuelas efectivas han indicado que la calidad de las relaciones profesorestudiante está en el corazón del proceso de aprendizaje. Por su parte, la investigación ha puesto de manifiesto que la mejora de las instituciones escolares está vinculada a los procesos de enseñanza-aprendizaje, en simultáneo con el desarrollo del profesorado. Lo que sucede dentro del aula es extremadamente importante para generar y mantener la mejora escolar. (HARRIS, 2002, p. 82)

Ha resultado ser un "lugar común" de quienes defendemos la importancia de la formación para el ejercicio profesional de la docencia comenzar nuestros argumentos señalando que, efectivamente, enseñar es una tarea compleja. Lo es más aún en los días que corren. Bastaría analizar, para convencerse de ello, las inmensas críticas que el profesorado, en general, realiza al referirse a su trabajo como docentes: que los alumnos llegan peor preparados, con menor motivación e intereses más dispersos; que las condiciones del trabajo se han ido haciendo cada vez más precarias; que las exigencias burocráticas les impiden desarrollar sus tarea prioritarias (docencia e investigación) con la tranquilidad necesaria; que los recursos disponibles son cada vez más escasos, etc. Y así sucesivamente en un proceso mitad protesta mitad catarsis. Ni qué decir tiene que, probablemente, tengan razón en muchas de esas quejas. Pero sirve de poco quedarse en la queja si no se profundiza un poco más en el problema para poder atisbar por dónde podría buscársele una 
solución.

Un aspecto común de todas esas quejas es el hecho de que el locus of control de la situación se coloca fuera de quienes las realizan. Así las causas del deterioro de la docencia universitaria, en la versión del profesorado, estarían siempre al margen de lo que los profesores hacemos. No es nuestra culpa, vendríamos a decir. La culpa es del gobierno que invierte menos, de los estudiantes que llegan peor preparados y carentes de la motivación y esfuerzo requeridos, del capitalismo que nos impone a todos unas reglas de juego al servicio de de las demandas empresariales. No está en nuestras manos resolver ese problema, venimos a decir. Somos las víctimas y no la causa de que las cosas no vayan tan bien como debieran en la Educación Superior. Ni qué decir tiene, una vez más, que hay mucha razón en tales consideraciones. Pero no toda. Hoy como siempre, la superación de los problemas de la formación depende en parte de las políticas y en parte del trabajo de los técnicos que las desarrollan. Precisamos, desde luego, de mejores políticas universitarias, pero precisamos, igualmente, de profesorado dispuesto a asumir sus responsabilidades.

Sato (1992), ingeniero paraguayo, presenta un esquema de cómo deberíamos abordar las cuestiones de la calidad de cara al establecimiento de pautas de mejora en la Administración y los Centros Educativos.

Existe, en primer lugar, la calidad de diseño que depende habitualmente de las gerencias, los gobiernos o quienes pueden tomar decisiones vinculadas a la financiación y los recursos. No es lo mismo construir una casa de protección oficial que una mansión faraónica, ni fabricar un coche de lujo o un utilitario. Cuando alguien diseña un producto o un proceso ya está incorporando una cierta idea de calidad que será la que marque las condiciones en que tal proceso o producto habrá de ser elaborado. La calidad del diseño está fuertemente vinculada al costo y las condiciones materiales (infraestructuras, equipamiento, materiales, etc.) y funcionales (personal, organización, sistemas de control, etc.). No se pueden exigir productos o procesos de alta calidad si el proceso de diseño estuvo orientado por consideraciones de baja calidad (baja inversión, escasos controles, bajo nivel de personal). En el mundo educativo, este tipo de contradicciones son frecuentes. Como señalaba críticamente James Wisdom, de la Agencia de Calidad SEDA del Reino Unido, en un reciente encuentro en Madrid: cuanto más se reduce la inversión más se elevan los estándares. Y eso es una contradicción. De difícil manera se van a lograr cotas elevadas de calidad en procesos que poseen una baja calidad de diseño. La institución universitaria y las políticas de Educación Superior deberían tenerlo en cuenta porque esta dimensión de la calidad va a condicionar todas las otras. 


\section{Miguel Zabalza Beraza}

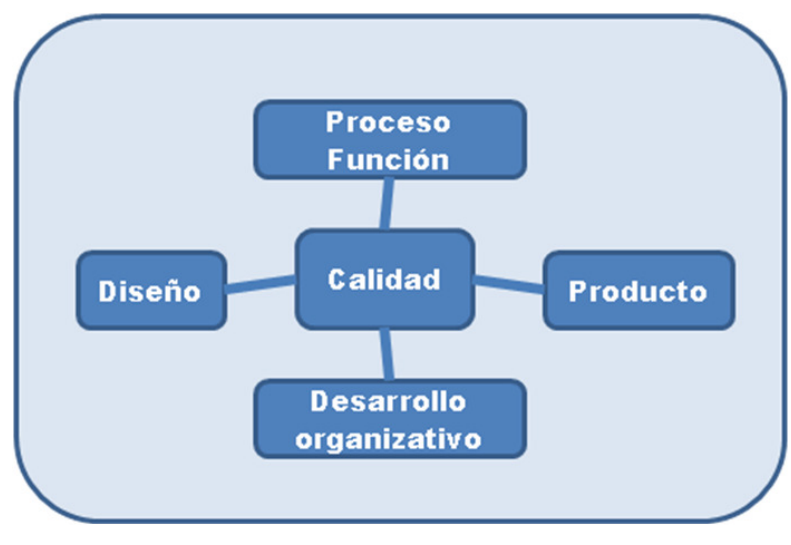

Gráfico 1: Componentes de una docencia de calidad

La calidad del proceso o función se refiere a los procedimientos a través de los cuales se pretende desarrollar la función prevista. En el caso de las Universidades, la formación de sus estudiantes, la investigación, etc. Si la calidad de diseño dependía básicamente de las gerencias (incluida la Administración Educativa), la calidad del proceso depende básicamente de los técnicos que los desarrollan. Claro que ellos y ellas pueden ver su trabajo condicionado por factores que no dependen de ellos pero, sin duda, el que el proceso salga bien también depende de ellos. Si hacen mal su trabajo, si no están bien preparados para desarrollarlo, si las decisiones que adoptan son incorrectas o las actitudes que mantienen son inadecuadas, con seguridad el proceso no se desarrollará en buenas condiciones.

El desarrollo organizativo es otra de las condiciones que enmarcan los procesos de calidad. Al igual que éstos, depende en buena medida de la calidad del diseño pero, también, de la buena organización de los procesos. Las tareas formativas que desarrolla la universidad no son compromisos individuales que asuma cada uno de sus profesores. Es un compromiso colectivo cuyo cumplimiento requiere de que toda la institución funcione adecuadamente y genere los dispositivos necesarios para que esos procesos formativos se desarrollen con eficacia. Como señalaba Zygmut Bauman, con frecuencia se nos exige buscar soluciones biográficas a problemas estructurales y contradicciones sistémicas. Es difícil llevar a cabo una docencia de calidad, imaginativa e innovadora si los Planes de Estudio no están bien planteados, si la dinámica de trabajo sigue basada en planteamientos individualistas, si se busca más dar respuesta a demandas burocráticas y de control que a procesos de calidad, etc.

Por su parte, la calidad del producto dependerá de que tanto la calidad de diseño como la calidad del proceso se hayan garantizado. La consecución de las finalidades institucionales (en nuestro caso la formación de nuestros estudiantes, los logros en investigación, el impacto en el desarrollo social y cultural de nuestro entorno) dependerá de que la universidad haya planteado en términos correctos tanto el diseño como los procesos. 
En definitiva, lo que quería resaltar con este excurso sobre la calidad es que el profesorado no puede plantearse al margen de la calidad de la docencia. Ni podemos plantear la calidad de la docencia al margen de nuestra capacidad para desarrollar dicha función de forma competente. $Y$ es en este contexto, donde podemos cerrar la tesis que pretendo desarrollar basada en tres puntos:

- La enseñanza universitaria es una actividad compleja y en la que se están produciendo cambios sustantivos que requieren de nuevos dispositivos tanto institucionales como organizativos y funcionales.

- Lograr una enseñanza de calidad en este contexto de complejidad exige disponer de profesorado capacitado y dispuesto a comprometerse tanto individual como colectivamente en ese empeño.

- Llegar a ser un profesor o profesora universitario cualificado requiere de una fuerte capacitación para el ejercicio de la docencia. Aunque la enseñanza tenga su parte de arte y se beneficie de las cualidades personales innatas de quien la ejerce, requiere, además, de competencias específicas que, como en otras profesiones, sólo se logran a través de una formación específica y especializada.

En esa misma línea de argumentación se mueve el documento de la OCDE que antes citaba. En él se venía a reclamar una convicción básica: los profesores cuentan. Se pretende, con ello, responder a la progresiva pérdida de protagonismo que ha ido sufriendo su figura y a las dudas que sobre su relevancia en el desarrollo de una docencia de calidad se han ido asentando en los últimos tiempos. En parte, reforzadas por el propio profesorado que en algunos casos se siente defraudado y descomprometido con la institución en la que trabaja.

Pese a ello, quien conozca la educación y se haya detenido a estudiarla sabe de sobra que no son las leyes, ni los políticos, ni los empresarios, ni los diversos fantasmas que recorren el subconsciente colectivo de unos y otros (el neoliberalismo, el Banco Mundial, el capital, la sociedad, o la religión) quienes pueden establecer el sentido último de la educación. Lo hacemos los profesores y profesoras que cubrimos el día a día del trabajo académico con los estudiantes. $Y$ si es bien cierto que los profesores no somos seres extraterrestres capaces de sustraernos a las influencias del medio social y cultural en el que vivimos y que, por tanto, las ideas vigentes de nuestro medio serán en buena medida nuestras propias ideas, no es menos cierto que somos nosotros quienes convertimos en acciones educativas las ideas y consignas. Tanto las que nosotros mismos generamos como las que vienen de las instrucciones políticas.

Han pasado ya 50 años desde que el Informe Coleman pretendiera demostrar que "las escuelas no hacen la diferencia" o dicho en otras palabras, que importa poco si se va a una escuela u otra porque buena parte de las variaciones de los resultados de la escolaridad pueden ser predichos antes de que un niño o niña entre a la escuela en función de las condiciones económicas, 


\section{Miguel Zabalza Beraza}

culturales o sociales de su familia y/o en base a sus propias características personales. Salvatis salvandis, un planteamiento similar se ha venido extendiendo en la forma en que algunos docentes viven la enseñanza en la universidad. Tuve un Rector que defendía convencido que la universidad no podía dar a un estudiante nada que él no fuera capaz de conseguir por sí mismo. Se hacía eco de aquel viejo adagio latino de que "quod Natura non dat Salmantica non praestat". No era tan importante, por tanto, la docencia. Lo eran más los recursos que se pusieran a disposición de los estudiantes: bibliotecas, laboratorios, infraestructuras, etc. Nuevamente las variables input como principales protagonistas de la calidad de los resultados formativos.

Afortunadamente, esa perspectiva determinista perdió vigencia y, aunque con ciertos ribetes utópicos, se ha considerado la institución escolar y la docencia como una importante condición para la obtención de buenos resultados formativos. No da lo mismo ir a una buena universidad que a una mala, no da lo mismo tener buenos que malos profesores, no se aprende igual con buenas o malas metodologías. Sólo desde esa convicción estaremos en condiciones de articular procesos formativos de calidad. Dicho en otras palabras, hemos de aceptar que los resultados de la Educación Superior vienen condicionados, desde luego, por variables que transcienden a la propia institución universitaria, pero resulta igualmente evidente que esos resultados van a variar, de manera sustantiva, en función de las propias características de las universidades a las que se asista y del profesorado con el que se tenga la fortuna (o desgracia) de aprender.

Y aceptado eso, se hacen evidentes las consecuencias que de ahí emanan. Entre ellas, la importancia del profesorado. Como escribe Enguita, no sólo el factor fundamental de una buena educación es, tras el propio alumno, el profesor, sino que la efectividad de su función consiste en cosas que dependen casi exclusivamente de él o ella (Enguita añade a ello que se trata de cosas simples y obvias, aunque en ese asunto, que sean simples y obvias, podemos discrepar de su planteamiento). Esas cosas son aquellas que tradicionalmente se han identificado con la tarea de enseñar: "conocer a fondo lo que tiene que enseñar, saber estructurarlo y explicarlo, poder mostrar su sentido y utilidad, ser capaz de organizar una situación o un proceso de enseñanza y/o aprendizaje y lograr una mínima empatía con el alumno/a" (pág. 61).

Esto es, una buena Educación Superior requiere de buenas instituciones y las buenas instituciones las hacen los buenos profesores y profesoras. Sin duda, ésa es la certeza que ha puesto en marcha todo el actual movimiento hacía el reclutamiento, la formación y el mantenimiento de buenos profesionales docentes.

\section{El triple componente de la figura docente}

Tres aspectos básicos podemos considerar cuando nos aproximamos a la figura del docente: la dimensión personal, la dimensión profesionaly la dimensión laboral. 
1) La dimensión personal apenas si había sido tomada en cuenta, salvo como factor teórico inseparable de cualquier acción humana, hasta que los movimientos feministas han hecho especial hincapié en la importancia de las condiciones cotidianas en que se desarrolla la vida de las personas que ejercen la docencia. Sin embargo, de siempre se ha sabido que el factor personal es un elemento clave en la actuación de los docentes y que sus características de personalidad, empatía, experiencias personales o situación de vida acaban ejerciendo una influencia incontrolable en la relación con los alumnos y con los colegas. Pero, desde el punto de vista de la formación, se ha tomado poco en cuenta esa circunstancia, quizás porque las universidades y los centros de formación no hemos sabido muy bien como entrar en ese territorio personal sin romper los límites de la privacidad y el derecho a la intimidad. En los últimos años, la aparición de nuevas corrientes psicológicas como las que tienen que ver con la "psicología emocional" (COLEMAN 1996 y 1997), el "coaching" (CABERO, 2008; KISE, 2006), la "autoeficacia docente" (PRIETO, 2008; PRIETO y BERMEJO, 2005) etc. han vuelto a poner sobre el candelero la necesidad de atender a esta importante dimensión de la madurez y equilibrio personal y algunas técnicas específicas para poderlo hacer. De todas formas es dudoso que las instituciones de formación incorporen este tipo de planteamientos a sus propuestas formativas. No sabemos cómo hacerlo, aunque estemos convencidos de la importancia de esta dimensión personal en el desarrollo de la docencia.

2) La dimensión profesional es aquella a la que se le presta mayor atención puesto que constituye la esencia de lo que un docente es y debe hacer. No cabe duda de que la perspectiva profesionalsalvó a los docentes de la zona difusa y límbica del amateurismo vocacional en la que durante muchos años ejercieron su profesión. El ejercicio de una profesión plantea requisitos que, sin duda, han fortalecida la identidad de la tarea docente: una formación específica y de alto nivel; unos sistemas de selección; el reconocimiento de la autonomía en su trabajo; la formación permanente; la carrera profesional, etc. Con todo, los beneficios indudables de la profesionalización no están exentos de riesgos. Algunos han criticado que la profesionalidad haya derivado, en muchos casos en profesionalismo, esto es, la defensa corporativa, el estancamiento, la pérdida de la vocación docente convirtiendo la enseñanza en un trabajo más, el ejercicio profesional basado en el título más que en el dominio de las acciones a realizar. Especial atención se está prestando en éste ámbito al proceso de socialización de los docentes en las instituciones a las que se integran (MARCELO, 2005) y a la forma en que van construyendo su propio estilo docente (teorías del posicionamiento de Harré y Van Langenhove, 1999; teorías de la construcción de la identidad profesionalBarnett y Di Napoli, 2007; Henkel, 2000). En cualquier caso, la profesión docente, como cualquier otra profesión está abocada a fuertes procesos de cambio y a la necesidad de formación a lo largo de la vida. A ello nos referiremos en los puntos siguientes porque constituye uno de las condiciones básicas para la mejora de la calidad de la docencia. 


\section{Miguel Zabalza Beraza}

3) Otros de los factores básicos que constituye la identidad del profesorado y que modula y condiciona su actuación y permanencia en el trabajo es la dimensión laboral. Los profesores y profesoras son trabajadores que desempeñan tareas específicas en instituciones educativas bajo unas ciertas cláusulas contractuales. Las múltiples modalidades y las muy diversas características contractuales a través de las que se vincula el profesorado a las instituciones en que ejercen la docencia, no pueden dejar de ejercer una fuerte influencia en su compromiso y dedicación. El documento de la OCDE al que vengo haciendo referencia alude a esa cuestión de forma reiterada. Cualquier de los modelos actualmente vigentes (la funcionarización o el contrato) tienen sus pros y sus contras. Se dice del primero que prima la titulación y la situación de entrada de los candidatos (selección por la carrera) y descuida el ajuste necesario a la especificidad de los puestos que los candidatos seleccionados han de atender a lo largo de su carrera profesional. Se dice de la segunda modalidad (el contrato o selección en función del puesto) que facilita ese ajuste entre los candidatos seleccionados y el puesto a desempeñar pero que precariza el empleo y provoca mayor vulnerabilidad profesional. Se trata, por tanto de una cuestión muy importante en los enfoques que tratan de mejorar la docencia. Profesores en situación precaria, sobrecargados de horas para poder alcanzar un salario aceptable, en situación de interinidad permanente o bajo riesgo de perder su trabajo resultan, por lo general, profesionales débiles y poco motivados. Hay que garantizar unas condiciones mínimas, y equiparables a los profesionales de su categoría, que permitan ejercer el trabajo en condiciones dignas. Dicho esto, se ha de reconocer, igualmente, que el salario no es el factor principal en lo que se refiere a la motivación e implicación de los docentes. No son los profesores o profesoras mejor pagados quienes más motivados están por su trabajo ni los que mas se implican en él. Hay otros factores complementarios que ejercen mayor influencia (el reconocimiento, la constatación del éxito con sus estudiantes; el sentimiento de autoeficacia, etc.).

He analizado superficialmente las tres grandes dimensiones de la figura de los docentes. De ellas, la que tiene mayor relevancia, al menos para el objetivo de este texto, es la que se refiere a la dimensión profesional. A ella me referiré a continuación destacando aquellos aspectos que, desde mi punto de vista, constituyen los ejes de lo que ha de ser el cambio de nuestro trabajo de cara a mejorar la calidad de la docencia que llevamos a cabo. Y los que han de ser, por ende, los ejes sobre los que articular una formación docente orientada a la consecución de altos niveles de calidad en el aprendizaje de los estudiantes.

\section{II) Evolución de la figura del profesor}

Es claro que se está produciendo una reconstrucción de la figura del profesor. En todos los niveles del sistema educativo, desde la Educación Infantil a la Universidad. Aunque, a decir verdad, este cambio se nota, por ahora, más en el ámbito de la teoría y de las propuestas que en la realidad de los hechos. Pero es importante que quienes participamos en los procesos de formación de 
los futuros profesores y profesoras lo tomemos en consideración, siquiera sea como "orientación de base". En definitiva se espera que también nosotros, los formadores del profesorado, actuemos como agentes de ese cambio. Y debemos hacerlo en un doble sentido: hemos de cambiar nosotros mismos (que también somos profesores) y hemos de cambiar la forma en que formamos a quienes han de ejercer tareas de docencia en la Educación Superior.

Ese itinerario de cambio podríamos plantearlo en torno a 4 nuevas perspectivas. En algún sentido, son orientaciones comunes para todos los niveles educativos, pero para atenerme a la temática de este trabajo me referiré más específicamente a lo que se refiere a la formación de los docentes universitarios.

a) El retorno de una visión excesiva y erróneamente reductiva de la profesionalidad a una perspectiva de profesionalidad doble (científica y docente) y abierta a la dinámica cultural del propio entorno.

No quisiera dar pie a malinterpretaciones. En el fondo, la idea es sencilla. La universidad ha entendido tradicionalmente que quienes ejercían la docencia debían ser competentes en su espacio científico o profesional. Siendo que la docencia resultaba una variable opaca en el proceso, la relevancia mayor la tenían el currículum vitae de los candidatos y sus méritos científicos o profesionales. Esa visión reductiva de la profesionalidad aún se mantiene hoy día y permanece bastante blindada en la cultura universitaria y en los procedimientos de selección y promoción del profesorado. Para ser un buen docente basta ser un buen investigador o, en su caso, un buen profesional. Muchas personas, incluidos los responsables universitarios, siguen convencidas de este aserto. La nueva profesionalidad que se propone no pretende negar ésta. Resultaría absurdo y vacuo. Nadie puede enseñar lo que no sabe. Pero bien puede suceder que quien sepa mucho de alguna cosa no tenga las habilidades necesarias para lograr que sus estudiantes aprendan con él cosas que, a veces, tienen que ver con lo que él sabe, pero otras no.

La doble profesionalidad implica que los docentes han logrado complementar su espacio de dominio científico con otro perteneciente al dominio pedagógico. No basta con quejarse de los estudiantes actuales o de las dificultades para que accedan al conocimiento que pretendemos transmitirles. Puede que no sea cierto aquello de que "no hay malos estudiantes, sino docentes incompetentes", pero no puede extrañar que personas que nunca se han preparado para enseñar tengan dificultades para hacerlo. Más todavía si su objetivo no es, ni siquiera, enseñar (para lo que sería suficiente conocer bien la disciplina y tener ciertas dotes de comunicación) sino lograr que los estudiantes aprendan. "O noso profesor aprendeunos moito", se dice en gallego para indicar que nuestro profesor nos enseñó mucho. Su mérito no fue que enseñara/explicara bien, sino que logró que aprendiéramos. 


\section{Miguel Zabalza Beraza}

Una priorización excesiva de los componentes científicos o profesionales de la formación docente puede generar vacíos en otras dimensiones de su formación, sobre todo, en aquellos que afectan a su formación pedagógica y personal, pero también a su formación cultural. En el fondo, la competencia docente del profesorado universitario tiene que estar asentada en cuatro ejes fundamentales: el dominio de la propia disciplina o campo profesional; las competencias pedagógicas; la dimensión personal y el compromiso cultural y social.

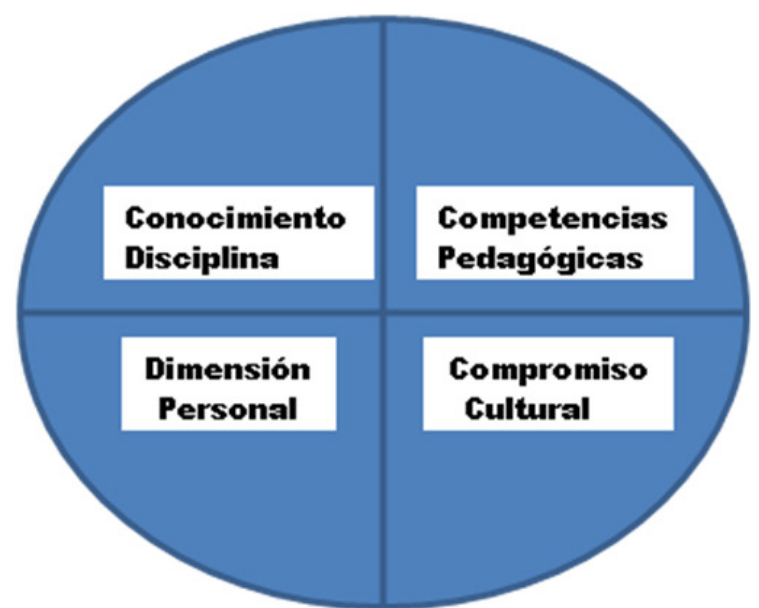

Gráfico 2: Dimensiones de la profesionalidad docente

Las dos primeras columnas de este edificio han sido suficientemente tratadas y argumentadas. Seguramente la dimensión personal plantea más difícil defensa, sobre todo en el ámbito universitario. De todas formas, resulta evidente en las investigaciones sobre la docencia, la importancia que adquieren las variables de tipo personal de los docentes. Aspectos como su capacidad de empatía, su disposición al diálogo, su apertura, su equilibrio personal, su simpatía, etc. juegan un papel fundamental en la configuración de contextos de aprendizaje amigables y eficaces. La cuarta de las dimensiones resulta más novedosa y, probablemente, nos llevará a replantear la formación de los docentes en el futuro. Ya hay grupos de universidades que se han consorciado para superar la tendencia excesiva a una formación únicamente técnica y profesionalizante: superar el training para alcanzar la education. La formación, para ser efectiva, ha de superar esa barrera tendiendo a que los sujetos formados no solamente sean poseedores de un título que los acredite como competentes, sino que hayan desarrollado todo un bagaje de conocimientos vitales que los vinculen a la sociedad a la que pertenecen (o al mundo en general) y les comprometan en su mejora.

El valor de la cultura, lo ha resaltado con mucho énfasis Bertagna (2003) como un nuevo marco de referencia para la formación de los docentes de la nueva educación que se ha ido abriendo camino en las reformas que han ido 
reconfigurando el panorama educativo internacional. En un momento de cierta confusión cultural con una notable pérdida de la identidad de las comunidades, con un predominio claro de la economía sobre la cultura, el papel de los profesores resulta fundamental. Como señala este autor, se trata de una visión de la cultura sustentada en una doble dimensión:

1. La cultura, en el espacio próximo, en lo que significa de conocimiento y reconocimiento de lo que nos es propio, de lo que nos ayuda a interpretar nuestro presente desde nuestro propio pasado. Ser capaces de entender una frase famosa, un cuadro, una producción musical, una danza; ser capaces de usar con propiedad la lengua materna, de poseer las coordenadas que nos permitan interpretar, críticamente, desde luego, nuestras costumbres, tradiciones, estilos de vida. Al final, una de las funciones básicas de la educación, desde la escuela infantil a la universidad, es capacitar a los estudiantes para integrarse en su grupo social y cultural. También para salir de él, si lo desean. En otros tiempos, ese proceso podría producirse de forma natural. Hoy en día, las presiones de la globalización son tan fuertes que se precisa de condiciones especiales para poder construirse ese marco de referencia cultural contextualizado que nos permita vivir en el mundo sin perder nuestra propia identidad cultural. La formación universitaria, que viene de universitas y hace resonancia a lo universal, no tiene por qué entrar en contradicción con lo más próximo y propio, ni la globalización para la que hemos de preparar a nuestros estudiantes entra en contradicción con el compromiso con lo local.

2. También la cultura en su dimensión más global, más humanística como la capacidad de pensar con una cierta profundidad sobre cuestiones que configuran las grandes problemáticas existenciales: la vida, la muerte, las relaciones humanas, la pobreza y la riqueza, la belleza, el dolor, la democracia, etc. En el fondo, no es otra cosa que aprender a desarrollar la capacidad más específicamente humana. Ser culto, más que saber de memoria muchas cosas, es ser capaz de plantearse los asuntos con profundidad, huir de la superficialidad, del mero activismo. Hay profesores a los que les produce un cierto reparo dar ese salto de los enunciados descriptivos de sus disciplinas a consideraciones de más hondo calado. Desde luego es un salto difícil de dar si uno mismo, si el propio docente no posee esa competencia cultural. De ahí que sea tan importante insistir en este punto cuando hablamos de formación del profesorado.

b) El paso del "experto en los contenidos de la disciplina" a experto en la "didáctica de la disciplina"

Tanto la legislación, como las orientaciones para las reformas curriculares y la literatura pedagógica vienen insistiendo durante los últimos años en este punto. Pero el sistema, al menos en algunos países sigue propiciando más la primera figura (la del experto en la materia) que la segunda (competente en su didáctica). Podríamos decir que se asume como indiscutible el viejo principio de que cuanto más sepas de algo mejor podrás enseñarlo. 


\section{Miguel Zabalza Beraza}

Sin embargo, como ha ido insistiendo la pedagogía francesa (y en especial los profesores de matemáticas) la tarea de enseñar que desarrollamos los profesores (eso es, en definitiva, la didáctica) se sustancia en tres grande operaciones:

1) La trasposición didáctica, esto es la conversión del savoir savant, el conocimiento de los especialistas, en savoir à enseigner, lo que en función de su desarrollo y sus capacidades pueden aprender nuestros estudiantes;

2) El contrato pedagógico, esto es el tipo de relación y las condiciones bajo las cuales se producirá la interacción entre profesores y estudiantes (entre cada profesor y cada estudiante);

3) La mediación didáctica, esto es, el tipo de contacto entre aprendiz y conocimiento que se propiciará en el proceso de aprendizaje.

Como puede constatarse, solamente la primera de dichas funciones, y sólo de forma parcial, depende de forma directa del nivel de expertía que se posea en el dominio de los contenidos curriculares. Enseñar tiene que ser, necesariamente, algo más que ser experto en una disciplina. Sin embargo falta todavía una clara articulación institucional que favorezca dicho proceso. Posiblemente se precise un trabajo de formación en el cual se produzca una mayor integración entre la Didáctica General (que entiende de metodologías y cuestiones generales pero no de contenidos específicos) y las Didácticas Específicas.

En el ámbito universitario la necesidad de esta complementación es aún mayor pues se posee menor experiencia. No existe una investigación suficientemente seria y mantenida sobre didácticas específicas en la Educación Superior. Por eso, la formación de profesorado, en los nuevos enfoques del lifelong learning, resulta tan importante en este momento. Formarse para la docencia no es solo asistir a cursos aislado sino desarrollar todo un proceso continuado de formación que permita a quienes lo deseen acceder a los niveles académicos más elevados: especializaciones, máster, doctorado, etc. Cuando tengamos muchos profesores y profesoras que hayan alcanzado su doble doctorado (doctores en su materia y doctores en la didáctica de su materia) podremos contar con un bagaje de conocimiento bien fundamentado sobre cómo se debe enseñar en la universidad.

Ése es el objetivo a medio plazo. En cualquier caso, este salto cualitativo que nos lleva de la expertía a secas en la propia disciplina a la competencia en su didáctica nos llevará necesariamente a hacer una mayor insistencia en las metodologías que en los contenidos, a dedicar mayor espacio a las prácticas (bien en formato de prácticas reales bien como simulaciones) que a la mera extensión de nociones conceptuales. Se van poniendo en marcha experiencias interesantes en este sentido. Los actuales modelos de enseñanza basados en 
competencias podrían abrir una interesante brecha en el logocentrismo vigente. Las competencias obligan a un trabajo coordinado, basado en adquisiciones a medio plazo y a través de influencias convergentes de las diversas disciplinas.

\section{c) Del "profesor de la clase" al "profesor del Centro"}

En este caso se trata de un cambio básico en la "cultura profesional" de los docentes. Su identidad profesional se centra habitualmente en lo "individual" y suele estar ligada a la materia que imparten y/o al curso que atienden: ser buen profesor suele significar saber mucho de la materia y saberla explicar a los alumnos de la clase. Pero de lo que se trata es de superar el individualismo para entrar en un sistema de trabajo cooperativo, a nivel local, y en red, a nivel general. No nos va a ser fácil.

Nuevas funciones y nuevas demandas se hacen ahora al profesor (más como miembro de un equipo docente que como profesor individual): hacer propuestas curriculares capaces de dar respuesta a las demandas diversificadas de sus estudiantes, integrar su trabajo formativo en las coordenadas que establece el Plan de Estudios, diseñar materiales didácticos que resulten atractivos y comprensibles, diseñar ambientes de aprendizaje ricos y estimulantes combinando lo académico y lo profesional, llevar a cabo la evaluación de sus estudiantes de manera coherente con las competencias a desarrollar. En fin, todo un conjunto de acciones que van más allá del habitual trabajo a solas en el aula. No digamos nada, si el proyecto institucional es claro y contiene líneas estratégicas determinadas: trabajo por módulos, metodologías activas, desarrollo de productos o trabajos, planteamientos interdisciplinares, etc. En definitiva se trata de procesos de toma de decisiones didácticas en las que cada profesor/a participa no como docente individual sino como miembro de un grupo y de una institución. Esto es realmente nuevo para muchos de nosotros.

Muchas de estas nuevas demandas sociales y del nuevo tipo de estudiantes que acuden a nuestras aulas van a exigir de las políticas universitarias planes de formación para el personal docente. Nuestras necesidades formativas (vinculadas muchas de ellas a la propia esencia de lo que es diseñar un currículum adaptado que exprese un "proyecto formativo integrado y original" para el propio centro y para los alumnos que acuden a él) aparecen como demandas básicas de los grupos de profesores más inquietos: cómo resolver el tema de la continuidad curricular; cómo llegar al consenso en la toma de decisiones; cómo diseñar, llenar de contenido y poner en marcha un proyecto institucional que vaya más allá de la mera yuxtaposición de asignaturas; cómo establecer estructuras de coordinación capaces de dotar de coherencia el trabajo conjunto; cómo dar respuesta a la progresiva heterogeneidad del alumnado y a su origen multicultural y multilingüístico; cómo abordar los problemas de rendimiento, como establecer sistemas de coordinación con el mundo del trabajo, cómo afrontar el abandono y/o el fracaso, etc. Todas esas cuestiones desbordan lo que podamos saber sobre nuestra materia y su enseñanza. Se trata de otro tipo de 


\section{Miguel Zabalza Beraza}

actuaciones más colectivas e institucionales para las que estamos escasamente preparados (porque tampoco los sistemas de formación insisten en ello).

Este aspecto objetivo y funcional del trabajo colectivo no debe hacernos olvidar la importancia psicológica que la dimensión actitudinal de nuestra profesión docente conlleva. Uno de los muchos problemas que minan la eficacia de los docentes es, justamente, el individualismo con que afrontan su trabajo y la soledad que ésta genera. El trabajo en equipo dota, sin duda, de una mayor seguridad y una perspectiva de conjunto más amplia. Muchos buenos docentes se sienten inseguros sobre si lo que están haciendo estará bien, sobre todo si constatan que los resultados que obtienen con sus estudiantes no son todo lo positivos que esperaban. El trabajo en equipo (y/o como veremos en el siguiente punto en red) genera ese espacio colectivo en el que poner en común experiencias gratificantes $\mathrm{y} / \mathrm{o}$ frustrantes y pedir a los colegas ayuda para afianzar las primeras y neutralizar las segundas. Por eso resulta improbable, por no decir imposible, el que mejore nuestra docencia si ésta sigue siendo concebida y realizada como una actividad individual y poco visible (y, por tanto, poco compartida). Como nos recuerdan Austin, Brocato y La Fleur (1993) a partir de los datos de su investigación, los profesores construimos nuestro rol docente y nuestro conocimiento profesional especialmente a través de la socialización: observación de otros docentes, feedback recibido de los colegas y alumnos, trabajo docente en grupo (o en red, como acabo de señalar), etc. Esa construcción del conocimiento profesional es un proceso personal que no tiene mucho que ver con la cantidad de práctica que hayamos desarrollado. El llegar a ser un buen docente no tiene relación con la mucha o poca práctica que se posea (AMUNSEN, GRYSPEERDT e MOXNESS, 1993) sino con la apertura al exterior y el contraste con las experiencias de los otros.

\section{d) La apertura a la globalización y al trabajo en red}

La globalización y la progresiva incorporación de las TIC al mundo de la enseñanza han abierto nuevas posibilidades y retos para el trabajo docente. Uno de los más importantes es, sin duda, la posibilidad de establecer conexiones interinstitucionales como mecanismo para la mejora de la enseñanza. Posibilidad que, sin embargo, está teniendo notables dificultades para asentarse. Probablemente pese en ello la cultura del individualismo tan preponderante en el ejercicio de la docencia. Parece poco probable que si los profesores tenemos problemas para colaborar con los compañeros de nuestra propia institución vayamos a hacerlo fácilmente con los de otras.

Los nuevos sistemas de correo electrónico, de intercambio de documentos (incluidas fotografías, vídeos, bases de datos, etc.), la posibilidad ya más sofisticada de llevar a cabo video-conferencias on line, establecer foros y paneles de discusión, etc. permiten formatos de intercambio muy útiles para el desarrollo de actividades docentes compartidas. En este sentido, las redes interinstitucionales, incluidas aquellas entre centros de diversos países, pueden desarrollar iniciativas muy interesantes para la docencia: 
- el establecimiento de programas, materiales didácticos y/o guías docentes similares para sus estudiantes facilitando así los intercambios y las interacciones entre ellos (el trabajo en red de los estudiantes puede resultar una nueva forma de aprendizaje entre iguales, peer learning);

- la creación de una factoría de recursos para la docencia (casos prácticos, problemas, textos, materiales didácticos de tipo gráfico o multimedia, recursos para la evaluación) que compartan los profesores encargados de la misma o similares disciplinas en diversas instituciones;

- ejemplos de buenas prácticas docentes o de iniciativas de innovación puestas en marcha por parte de alguno de los miembros de la red y que podrían ser replicadas por el resto de colegas;

- la disponibilidad de contextos naturales, sociales o culturales diversos que los estudiantes podrían usufructuar para la realización de sus trabajos (por ejemplo recoger, analizar y comparar actuaciones profesionales, legislación, tradiciones culturales, estudios de campo, etc.);

- en el caso de instituciones de países en vías de desarrollo, instituciones pequeñas, jóvenes o menos dotadas, la red puede proporcionar un tipo de recursos que la propia institución no estaría en condiciones de disponer por sí misma.

En este sentido, las redes de docencia y/o para la docencia (en su más amplio sentido e incluyendo, por tanto, las redes de bibliotecas, de recursos multimedia, etc.) pueden jugar un enorme papel facilitador del trabajo de los docentes. Y, en consecuencia, en la mejora de la calidad de la docencia que impartimos.

\section{III) Evolución en los procesos de "formación de profesores"}

También en este apartado quisiera hacer una rápida mención a algunas de las variaciones habidas en los planteamientos de base de la formación de profesores.

a) De la formación dirigida a "la movilización" a la formación dirigida al "desarrollo práctico de procesos". O dicho de otra manera, de los "congresos" o "cursos" a los "grupos de trabajo".

En cualquier proceso de formación resulta necesaria una fase inicial de motivación y movilización. Por ejemplo, las conferencias, cursos de corta duración, congresos, etc. sin importantes recursos para la movilización. A través de ella se aportan mensajes de estimulación al cambio, se hacen panorámicas generales de la situación o de los temas a trabajar, etc. 


\section{Miguel Zabalza Beraza}

La movilización posee características comunicativas y metodológicas propias: puede hacerse a grandes grupos, exige periodos de corta duración, el formato curso resulta suficiente, el protagonista principal de los intercambios es el charlista (generalmente es él quien expone, anima y trabaja mientras el grupo receptor escucha y se motiva). La función de quien da la charla o el curso es hacer un discurso atractivo (no necesariamente práctico), seducir al auditorio de manera que se sientan motivados e interesados en el tema, etc. A mí me ha tocado muchas veces hacer ese papel que resulta necesario pero es insuficiente.

Una formación dirigida al desarrollo práctico de procesos presenta características muy diferentes. La principal de ellas es que el protagonismo del trabajo se traslada al grupo en formación. Y ello trae como corolario cambios profundos en la estructura del proceso formativo en sí. Me atrevería a citar algunos de los que yo mismo he vivido como más significativos:

- en primer lugar que el formato conferencia o curso ya no es útil (no vale que vengan especialistas a soltar cada uno su rollo por muy bien que sean capaces de hacerlo). Se requiere entrar en formatos tipo taller, grupo de trabajo, etc.;

- en segundo lugar, eso varía la naturaleza del propio formador. Ya no resulta tan interesante que sea alguien ducho en la "teoría de" sino alguien experimentado en la "práctica con". Por ejemplo, si el grupo quiere saber sobre cuestiones generales referidas a los materiales didácticos quien sepa de eso lo puede explicar; pero si su demanda se refiere a la "construcción de" o a "buen uso de", está claro que es más propio que se trabaje con quien tenga experiencia en desarrollar materiales didácticos y usarlos en un aula;

- en tercer lugar, varía el "tiempo". Cualquier proceso de reconstrucción práctica exige formatos temporales extensivos y no intensivos.

- pero, sobre todo, varía el papel que juega el propio grupo de formandos. Desarrollar procesos prácticos es "trabajar" y eso lleva implícitos toda una serie de fases y componentes en los cuales se ejerce un papel "activo" en tanto que protagonistas del proceso. Eso, a su vez, implica mayor "sufrimiento" y "disponibilidad al riesgo" por parte de los participantes (el salto del "escuchar complacido" al "hacer titubeante" es muy duro, y con frecuencia se producen alianzas y resistencias para retrasarlo o, incluso, evitarlo).

En cualquier caso, esta evolución en el proceso formativo me parece fundamental. Sin duda alguna es preciso iniciar la formación con actuaciones destinadas a la movilización. La experiencia en formación del profesorado universitario nos ha enseñado que aquellos docentes que, por cualquier circunstancia inician algún proceso de formación, acaban repitiendo y son quienes más formación demandan. Es decir, la formación, en cualquiera de sus formatos, ejerce realmente ese papel movilizador. El problema reside cuando esa fase inicial se enquista y el sistema formativo no acaba de salir de ella. En esos 
casos la teoría, el "mundo del hablar" se adueña del escenario y la formación acaba reduciéndose a una siembra masiva de nuevas nociones y conceptos con escaso impacto sobre las prácticas docentes reales.

referir.

Y esto nos sitúa frente al segundo de los dilemas a los que me quería

b) La transformación piagetiana: de la formación dirigida a "la asimilación" a la formación dirigida a "la acomodación".

Traigo a cuento aquí, no sé si de manera suficientemente ortodoxa, los dos conceptos piagetianos: "asimilación" se refiere a la incorporación de nuevas informaciones a nuestros esquemas; "acomodación" se refiere a cuando nuestros esquemas previos se modifican y reestructuran en función de la nueva información.

Como es bien sabido el auténtico aprendizaje está vinculado a la reestructuración de esquemas previos (esquemas conceptuales y operativos) en base a nuevas informaciones y experiencias. Planteado este dilema a los procesos de formación de profesores volvemos a estar en una contradicción similar a la mencionada en el punto anterior: los formatos de formación habituales están, en general, más orientados a la "asimilación" que a la "acomodación". Con mucha frecuencia los asistentes a cursos y grupos de formación regresan a sus casas con la cabeza llena de nuevas ideas, con un cierto empacho conceptual (¡han sido tantas cosas tan diferentes dichas en tan poco tiempo...!) pero con escasas propuestas para ir transformando su pensamiento y su acción docente en base a ellas.

Los llamados "modelos culturales de formación" suelen reflejar esa tendencia a la asimilación. Dicen de ellos que son los modelos de las tres "c": conceptualismo, contenidos, centralismo (la oferta formativa no surge de la demanda explícita sino de lo que es interpretado por necesidad desde la Administración educativa o desde las diferentes agencias de formación).

Desde mi punto de vista, las principales condiciones que plantea la acomodación son el tiempo y la práctica. Se precisa tiempo para ir asentando las ideas, y práctica para irlas consolidando, para ir reorganizando nuestra situación mental. Una buena acomodación no se puede hacer sin tiempo, se trata de procesos que no se pueden acelerar. Obviamente el tiempo sólo no es suficiente. También es preciso el contraste de las nociones a través de oportunidades de aplicación de las mismas (para ver si funcionan o no en la práctica y cómo lo hacen, si resultan útiles en el propio contexto, si plantean nuevas necesidades, etc.).

En definitiva, también en el caso de los programas de formación (como en cualquier proceso de aprendizaje) los momentos de"asimilación" han de ir 


\section{Miguel Zabalza Beraza}

seguidos de momentos de "acomodación". Y la acomodación en estos casos significa sobre todo "práctica guiada". El gran problema suele residir en que, en muchos casos, tampoco los formadores de profesores han hecho ese itinerario, también ellos y ellas están, con respecto a las cosas sobre las que forman, en el momento de la asimilación: dominan los conceptos pero no tienen experiencia en ponerlos en práctica. Son eruditos pero no competentes, funcionan como "expertos" más que como "experimentados". No siempre sucede eso, claro está. Hay formadores y formadoras muy experimentados, afortunadamente.

\section{c) La aparición de nuevos modelos de formación.}

No son, en realidad, nuevos pero sí van adquiriendo un nuevo sentido e importancia de cara a articular la respuesta que se puede ofrecer a las demandas de formación del profesorado. En muchos casos tales demandas presentan dos dimensiones más resaltables: la demanda de respuestas técnicas a los problemas profesionales y la demanda de apoyo personal en general (ligada a la situación de estrés, de desorientación, de soledad en el trabajo, etc.).

Al primer tipo de demandas responden bien los modelos "técnicodidácticos". Suelen estar orientados al desarrollo de procesos de índole metodológica o bien a la elaboración de recursos. En su desarrollo resulta fundamental tomar en consideración lo señalado en los puntos anteriores. Es preciso también ajustar el contenido práctico del curso a las necesidades a las que se pretende dar respuesta y a la especificidad de los contextos en los que virtualmente se espera poner en práctica el trabajo desarrollado. Por ello, el análisis del contexto y la evaluación de las necesidades forma parte del propio proceso de formación. Tienen más sentido de taller que de curso y un formato más extensivo y gradual que intensivo.

Una orientación distinta tienen los modelos "psico-sociales". En ellos se trata de dar respuesta a demandas más indefinidas y que tienen relación con la propia identidad profesional y la naturaleza institucional de nuestro trabajo. El formato habitual suelen ser los "grupos de formación" en los cuales se analizan las percepciones, vivencias, pautas de actuación de los asistentes, se realizan análisis institucionales de los propios escenarios profesionales y se planifican conjuntamente procesos de intervención cuya realización posterior es igualmente analizada por el grupo. El modelo de cambio es, en este segundo caso, más "cultural" (se trata de iniciar el cambio por uno mismo, por la forma de afrontar la institución, el trabajo, las relaciones, los enfoques) que "técnico" (aunque, ciertamente, a lo largo del trabajo en el grupo de formación se van abordando y trabajando las nuevas técnicas que se pretenda poner en marcha para llevar a cabo los "proyectos" de intervención generados en el grupo). Quizá lo más notable de estos grupos sea la reconstrucción de lo "colectivo" como escenario prioritario para el desarrollo profesional. 
d) Nuevas líneas básicas de desarrollo de la formación del profesorado: el aprendizaje a lo largo de la vida y la transferencia de créditos.

Guasti (1990) ofrecía hace ya años un esquema de cuatro puntos para resumir su análisis de cuáles podrían ser los ejes del cambio en los modelos de formación: plina).

1) formación centrada en la clase (frente a la centralidad de la disci-

2) refuerzo de los vínculos entre formación - investigación.

3) diferenciación de las funciones (en la medida que van apareciendo nuevos roles en el ejercicio profesional).

4) red de servicios de apoyo.

Hoy en día, las cosa han ido evolucionando pero estos 4 puntos siguen siendo muy relevantes en los actuales enfoques sobre la formación. El primero de los ejes conecta casi literalmente con consideraciones hechas en puntos anteriores, aunque quizás estaría mejor definido si en lugar de decir "centrada en la clase" dijéramos "centrada en el aprendizaje de nuestros estudiantes" (insistiendo en lo de "nuestros", no en unos estudiantes imaginarios y que se acomodaran bien a nuestras expectativas).

La conexión enseñanza - investigación resulta uno de los "leimotivs" habituales en los programas de formación, aunque no siempre acaban convirtiéndose en operaciones y estrategias concretas. En mi opinión la actual insistencia en actuaciones reflexivas no siempre logra conectar con el auténtico sentido de la conexión enseñanza - investigación como eje del desarrollo profesional. Con frecuencia reflexionar se reduce a "hablar y opinar" más que a constatar y contrastar sistemáticamente las actuaciones profesionales y los supuestos de los que parten.

La diferenciación de funciones se va haciendo cada vez más necesaria en el interior de las instituciones formativas. Tal diferenciación se produce tanto en sentido vertical (en lo que se refiere al trabajo dentro de las propias titulaciones y áreas disciplinares de forma que se mantenga la necesaria continuidad y progresividad) como en sentido horizontal (en lo que se refiere al desarrollo de la coordinación entre programas y el desarrollo de las competencias transversales). Éste es el gran desafío de la nueva profesionalidad docente y lo es, por tanto, de los procesos formativos que la nutren: formarse para el trabajo en grupo y/o en red (clusters de profesores que imparten clusters de asignaturas), el diseño curricular por módulos, los procesos formativos en alternancia, etc.

Finalmente, el cuarto punto recogido por Guasti da mucho juego y conecta muy bien con los enfoques del lifelong learning. La idea del aprendizaje 


\section{Miguel Zabalza Beraza}

a lo largo de la vida resulta de una gran fuerza desde el punto de vista de la formación. No, precisamente, porque se refiera a cosas desconocidas o revolucionarias. Todo el mundo es consciente de que un ejercicio profesional responsable requiere de una formación continuada. No es suficiente un título o una acreditación. Se precisa una renovación constante al socaire de los cambios que se van produciendo en el mundo de las profesiones y de las tecnologías que en ellas se emplean. Pero la fuerza del lifelong learning reside, en mi opinión, en lo que ese planteamiento supone de exigencia institucional. la siguiente definición $n^{4}$ :

El grupo ELLI (The European Initiative on Lifelong Learning ${ }^{3}$ ) asumió

Llamamos "aprendizaje a lo largo de la vida" al desarrollo del potencial humano de las personas a través de un proceso de apoyo constante que estimule y capacite a los sujetos para adquirir los conocimientos, valores, habilidades y comprensión de las cosas que van a necesitar y para saber aplicarlos con confianza, creatividad y gozo en cuantos roles, circunstancias y ambientes se vean inmersos durante toda su vida. ${ }^{5}$

Lo importante de esta definición es lo bien que enlaza con las ideas antes expuestas en torno al sentido y valor de la formación porque la plantea como algo:

- que abarca todas las dimensiones del desarrollo humano (desde los conocimientos a los valores, pasando por las habilidades y los significados).

- que resalta la necesidad de un ecosistema particular (por eso se habla de "cultura del aprendizaje") en el que la formación no responde únicamente a una exigencia social y académica vinculada a una edad y una institución determinada sino que se trata de algo que se proyecta a lo largo de toda la vida y que precisa de sistemas de apoyo (infraestructuras, oportunidades, incentivos, etc.) que lo estimulen (a continuously supportive process, reza la definición).

- que busca, desde los inicios, educar a los sujetos en la puesta a punto de sus propios recursos y en el trabajo autónomo. Ciertas fórmulas de formación generan sobre todo dependencia y no autonomía. Hay que preparar a los docentes a gestionar su propio aprendizaje, al igual que deberán hacerlo ellos mismos con sus estudiantes (ése es el sentido del empowering individuals que se señala en la definición).

- que incorpora como contenidos de la formación aspectos valiosos para los diversos cometidos que los sujetos hayan de afrontar a lo largo de la vida (no solamente para dar respuesta a las exigencias académicas).

- que vincula la formación no solamente a la presión social y al sufrimiento personal (idea coherente con el viejo principio de que la letra con 
sangre entra o con el más actual de la presión a través de la evaluación y el meritoriaje) sino a la autoestima y el gozo personal.

En definitiva, la formación continua del profesorado universitario debe estar orientada a posibilitar un mejor ejercicio de la función que está llamado a ejercer. Pero se plantea tanto como una propuesta a los individuos y como una exigencia a las instituciones que deben crear los dispositivos y mecanismos necesarios para que esa posibilidad de formación sea real. De ahí que cada día vayan adquiriendo mayor sentido y relevancia institucional los denominados servicios de apoyo al profesorado. Servicios que pueden presentar diversos formatos y actuar según diferentes modalidades de apoyo. Se trata de que el profesorado tenga la oportunidad de ir progresando en su carrera docente y que disponga para ello de los recursos y oportunidades que pudiera necesitar.

Por eso la oferta formativa de las instituciones de Educación Superior para su propio profesorado no pueden limitarse a una serie de cursos aislados sino que debe constituir un Plan de Formación con ofertas diferenciadas según el nivel de exigencia o desarrollo profesional de quienes la demanden. Muchas universidades se están ya planteando propuestas formativas de 2 o nivel, esto es, iniciativas que resuelvan las demandas de aquel profesorado que ya está formado, que ya realizó los cursos básicos y obtuvo las correspondientes acreditaciones. Estas personas, cada vez más numerosas y con mayor nivel de exigencia, precisan de nuevas oportunidades de formación a su medida: bibliografía especializada, factorías de recursos on line, grupos de investigación sobre temas docentes, programas de movilidad interuniversitarios, másters de didácticas específicas en su propio campo, segundos doctorados, etc.

Y aquí es donde podemos introducir la transferencia de créditos de formación como una estructura de niveles que van permitiendo al profesorado más comprometido ir progresando en su carrera profesional docente. En este momento, ésa es la opción que hemos asumido un grupo de 20 universidades de diversos países a través del diseño de un máster internacional en docencia y gestión universitaria. La idea es que el esfuerzo formativo que muchos profesores han ido realizando a través de cursos pueda rentabilizarse para alcanzar un título de máster y éste, a su vez, suponga la base de un posible doctorado (o segundo doctorado, en su caso) para los docentes que lo deseen. El esquema de desarrollo sería el siguiente. 


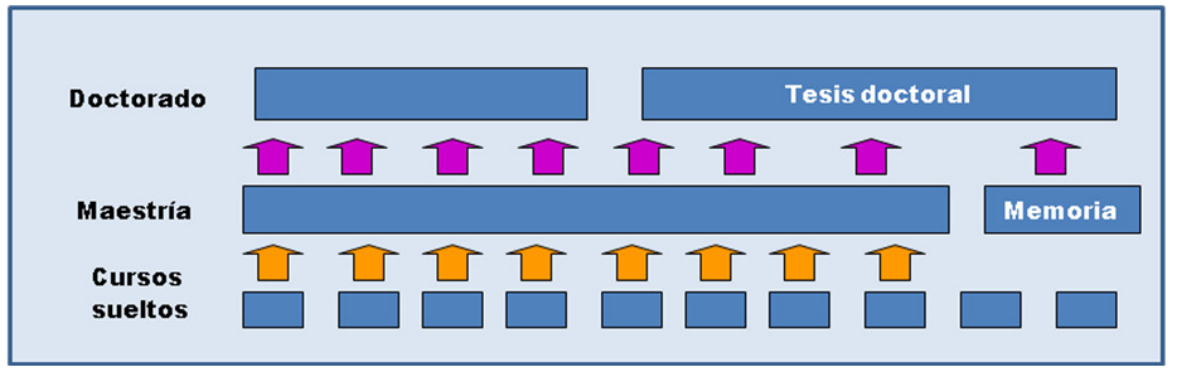

Gráfico 3: Diseño de la formación mediante transferencia de créditos

La idea es simple. La formación puede plantearse desde su inicio como un plan bien diseñado y coherente. En ese caso ya no hay problema porque la continuidad y progresión está establecida desde el inicio. Pero lo normal no suele ser así. Por lo general, las universidades, al menos en España, hacen una oferta variada de cursos que el profesorado va realizando o no según su particular interés. Pero esos cursos se agotan en sí mismos. No constituyen peldaños hacia un nivel superior o una acreditación académica reconocida. Ahí es donde se considera la transferencia de créditos (al igual que sucede en las titulaciones universitarias a través de los créditos ECTS). Los créditos logrados en los cursos individuales se aplican al siguiente nivel, que incluye otros así como las correspondientes exigencias de realización de una Memoria o tesis. De esta manera, la formación siempre cuenta con un horizonte a medio-largo plazo que le da sentido. No se agobia al profesorado con compromisos formativos excesivamente largos y exigentes (como pudiera suponer un máster en docencia o un nuevo doctorado) pero se les permite acceder a ellos a través de los pequeños pasos que puedan ir dando.

\section{Epilogo}

Hace unos años, Andy Hargreaves en la apertura de un congreso analizaba el tema de las emociones en la enseñanza. Ése es, también, el tema de uno de sus libros. Los profesores y la forma en que construimos nuestro papel y concebimos nuestra actuación nunca está exenta de emociones. Emociones que se alimentan de ideas, sensaciones, estereotipos, vivencias personales o referidas por otros, especialmente nuestros colegas y nuestros estudiantes. Al final, nuestra profesionalidad y nuestros conocimientos nunca son una cuestión puramente técnica (lo que sabemos sobre la disciplina y la forma de enseñarla) sino muy personal.

Muchos profesores han pasado por diversas reformas o intentos de cambio en la enseñanza. Para ellos cualquier propuesta de futuro se contrasta, necesariamente, con la memoria del pasado. Por eso, cómo nos sentimos en relación al pasado resulta muy importante. Algunos viven el síndrome del "cambio 
repetitivo" y eso los convierte en cínicos y/o en objetores pasivos de las propuestas de cambio: se dedican a sus clases y no quieren saber nada de los nuevos discursos.

En muchos casos la memoria del pasado se convierte en nostalgia. Hargreaves insistía en la importancia de la nostalgia como elemento clave en el quehacer docente. A veces se idealiza el pasado (la falsa nostalgia) y esa añoranza acaba cortocircuitando los procesos de mejora. En España, este fenómeno se ha convertido en un estereotipo social casi inexpugnable: todo era mejor en el pasado. Los alumnos estaban más motivados, aprendían más, estaban más apoyados por las familias, eran menos heretogéneos, etc. El pasado se convierte así en una contra-reforma que conlleva mucho desgaste emocional y resta energía para el progreso y la experimentación de nuevas alternativas.

Pero la nostalgia existe. La buena y la mala. Y se alimenta de emociones. Por eso el trabajo con profesores y el desarrollo de innovaciones requiere de actuaciones que vayan más allá de los elementos técnicos y cubran también ese espacio emocional. Es preciso partir de la particular resonancia que tenga la experiencia vivida y reactualizar (a veces desaprendiendo y rompiendo tópicos) los parámetros en los que se mueve nuestra forma de ver el papel de la escuela y de nuestra profesión. Al final, como ha titulado uno de sus libros Christopher Day, lo que mueve a los buenos docentes es la "Pasión por enseñar".

\section{Referências}

AUSTIN,A. E.; BROCATO, J. L.; LA FLEUR, M. "Learning to teach. The socialization of university teachers". Paper presented at the Annual Meeting of the American Educational research Association (AERA). Atlanta, GA. 1993.

AMUNDSEN, C.; GRYSPEERDT, D. E. MOXNESS, K. "Practice-centered inquiry: developing more effective teaching", Review of Higher Education, 16, 329353. 1993.

BARNETT, R. and DI NAPOLI, R. Changing Identities in Higher Education. London: Routledge. 2007.

BAUMAN, Z. La sociedad individualizada. Madrid: Cátedra. 2006.

BERTAGNA, G. "La formazione degli insegnanti nella scuola della reforma: il nuovo quadro di riferimento", en Analli dell'Istruzione XLXIX (1/2), p. 5-26, 2003.

CABERO, M. Coaching emocional. Barcelona: UOC// Kise, J. A (2006).: Differentiated Coaching:AFramework for Helping Teachers Change. Thousanks Oaks, Cal: Sage Publications. 2008.

CHEVALLARD, Y. La transposition didactique. Du savoir savant ao savoir enseignè. Grenoble: La Pensèe Sauvage. 1991.

COLEMAN, J. S. (Coord.). Equality of Educational Opportunity Study. http:// dx.doi.org/10.3886/ICPSR06389. 
COLEMAN, D. Inteligencia Emocional. Barcelona: Kairos.// (1999): La práctica en la inteligencia emocional. Barcelona: Kairos. 1996.

FERNÁNDEZ ENGUITA, M. Los profesores cuentan, en Revista de Educación, 340, 2006, p. 59-65.

GUASTI, L. Le stategie delle formazione in servizio. En Varios: Sistema educativo: prospettive di mutamento. Franco Angeli. Mòdena. 1990, p. 193203.

HARRIS, A. School improvement. What's in it for schools? London: RoutledgeFalmer Press. 2002.

HARRÉ, R., LANGENHOVE, L. The Dynamics of Social Episodes. Edited by H. Harré R., L. Langenhove. Positioning Theory: Moral Contexts of Intentional Action. Oxford: Blackwell, 1999.

HENKEL, M. Academic Identities and Policy Change in Higher Education. London: Jessica Kingsley, 2000.

LENOIR, Y. "Médiation cognitive et édiation didactique", en RAISKY, C.; CAILLOT, M. (Edis.) (1996): Au-delà des didactiques, le didactique. Débats autour de concepts fédérateurs. Bruxelles: De Boeck \& Larcier. p. 224-251, 1996.

MARCELO, C. Teachers learning for a learning society - literature review. In J. M. Moreno (Ed.), Learning to teach in the knowledge society. Final report (p. 234). Washington: World Bank. 2005.

PRIETO NAVARRO, L. La Autoeficacia del profesor universitario. Eficacia percibida y práctica docente. Madrid: Narcea. //Prieto Ursúa, M. y Bermejo Toro, L. (2005) : Malestar docente y creencias de autoeficacia del profesor, en Revista Española de Pedagogía (63) n. 232, 493-510. 2008.

RAISKY, C.; CAILLOT, M.: Au-delà de les didactiques, le didactique. Bruxelles : De Boeck. 1996.

SATO, K. La calidad en la buena administración. Uruguay: Ministerio de Industria, Energía y Minería. 1992.

\footnotetext{
Notas

${ }^{1}$ Puede consultarse, por ejemplo, el informe coordinado por Buchberger, P; Campos, B.P; Kallos, D y Stephenson, J.: Green paper on teacher Education in Europe. High Quality Teacher Education for High Quality Education and Training. Thematic Network on Teacher Education in Europe.TNTEE Editorial Office SE-90187 Umea Sweden Umea University. También la obra de SANDER, Th. (Edit.): Current changes and challenges in European Teacher Education. European Yearbook of Comparative Studies in Teacher Education 1994. Edit. Nijs-Herent. Bruxelles.

2 Teacher Education in Europe. An ETUCE Policy Paper. http://etuce.homestead.com/ Publications2008/ETUCE_PolicyPaper_en_web.pdf
} 
Formación del profesorado universitario: mejorar a los docentes para mejorar la docencia

${ }^{3}$ Existe una organización gemela a nivel mundial: la WILL (World Iniciative on Lifelong Learning). Ambas Iniciativas llevan a cabo, en sus respectivos ámbitos de competencia, la misión de promocionar los principios y conceptos derivados de los nuevos enfoques en formación continua.

${ }^{4}$ LONGWORTH, N. (s/f): "Lifelong Learning and the Schools: into the 21st. Century", en Longworth, N. \& Beernaert, Y. (Edits.): Lifelong Learning in Schools:an exploration into the impact of Lifelong Learning on the Schools Sector and on its implications for the Lifelong Learning needs of Teachers". ELLI (European Lifelong Learning Iniciative). Bruxelles. p. 4-16

${ }^{5}$ La definición original fue la siguiente: LIFELONG LEARNING is the development of Human potential through a continuously supportive process wich stimulates and empowers individuals to acquire all the knowledge, values, skills and understandings they will require throughout their lifetimes and to apply them with confidence, creativity and enjoyment in all roles, circumstances and environments.

\section{Correspondência}

Miguel Zabalza Beraza - Avda. Coroña, n. 2, CEP 15701, Santiago de Compostela, Espanã.

E-mail: miguel.zabalza@usc.es

Recebido em 02 de maio de 2011

Aprovado em 03 de junho de 2011 